\title{
Radiative and Leptonic $B$-meson Decays from the B-factories
}

John Walsh ${ }^{\mathrm{a}}$, on behalf of the BABAR and Belle Collaborations

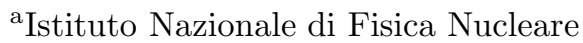

Largo Pontecorvo, 3

Pisa, Italy

Radiative and leptonic decays of $B$-mesons represent an excellent laboratory for the search for New Physics. I present here recent results on radiative and leptonic decays from the Belle and BABAR collaborations.

\section{Radiative Penguin and Leptonic $B$ - meson Decays}

Radiative penguin decays of $B$-mesons, in which a $b$ quark transitions to an $s$ or $d$ quark accompanied by either a photon or a pair of charged leptons (Fig. 1), are a sensitive probe of New Physics (NP) beyond the Standard Model (SM). These flavour changing neutral current decays are forbidden at tree level in the SM and hence strongly suppressed. In many NP scenarios these decays appear at the one-loop level, i.e., at the same order as the SM processes. Hence, the contributions of NP to branching ratios and asymmetries can be as large as the SM contributions, making these decays a good hunting ground for New Physics.
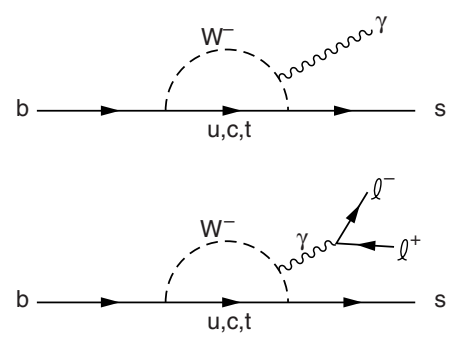

Figure 1. Feynman diagrams for radiative penguin processes. Additional diagrams with $Z^{0}$ and $W^{ \pm}$propagators not shown.

Leptonic $B$-meson decays, in which all final state particles are either charged leptons or neutrinos, are also often sensitive to the presence of
New Physics. As with the radiative penguin decays, virtual heavy particles predicted by NP can contribute to leptonic decays.

In this report, I discuss recent measurements of radiative penguin and leptonic $B$-meson decays performed at the BABAR [1] and Belle [2] experiments.

\section{Inclusive $B \rightarrow X_{s} \gamma$}

The branching fraction of the Standard Model inclusive $B \rightarrow X_{s} \gamma$ process is calculable to a precision of around $8 \%[3]$ :

$$
\left.\mathcal{B}\left(B \rightarrow X_{s} \gamma\right)\right|_{E_{\gamma}>1.6 \mathrm{GeV}}=(3.15 \pm 0.23) \times 10^{-4},
$$

where the rate is computed above the conventional lower limit of $1.6 \mathrm{GeV}$. The good precision of this calculation makes this process a powerful one in the search for NP and considerable experimental efforts have been put into making precise measurements of the inclusive rate.

Inclusive measurements are generally experimentally more difficult than reconstructing exclusive states: the lack of kinematic constraints leads to large backgrounds that require careful treatment. Recent measurements of $B \rightarrow X_{s} \gamma$ have relied on reducing the background from light quark events by tagging the other $B$-meson in the event, either by reconstructing it fully [i]] or by identifying the lepton coming from a semi-leptonic decay [5].

Ideally, one would like to measure the branching fraction over the full photon energy range, but experimental considerations require a minimum photon energy, generally in the neighborhood of 
1.8-1.9 GeV. Since the extrapolation down to 1.6 $\mathrm{GeV}$ introduces some model-dependence, experimentalists strive to keep this photon energy requirement as low as possible.

Belle has recently reported a new preliminary measurement of $B \rightarrow X_{s} \gamma$, based on $605 \mathrm{fb}^{-1}$ of data, where they do not require any sort of tagging of the other $B$-meson [6]. The advantage of this approach is the large gain in efficiency. The large backgrounds from continuum (light quark) events are suppressed using two Fisher discriminants, which exploit the topological differences in signal and background decays. Additional background suppression is achieved by directly reconstructing and vetoing $\pi^{0} \rightarrow \gamma \gamma$ and $\eta \rightarrow \gamma \gamma$ decays.

The remaining continuum background is estimated by analyzing data taken off-resonance, where no $B$-meson decays are present. Backgrounds from $B \bar{B}$ events are estimated using simulated events. Studies of control samples of real data allow for the correction of the Monte Carlo estimates for the major contributions to the $B \bar{B}$ backgrounds.

The resulting background-subtracted photon spectrum for $B \rightarrow X_{s} \gamma$ decays is shown in Fig. 2. The partial branching fraction, measured in the range $1.7<E_{\gamma}<2.8 \mathrm{GeV}$, where $E_{\gamma}$ is measured in the B-meson rest frame, is found to be

$\mathcal{B}\left(B \rightarrow X_{s} \gamma\right)=(3.31 \pm 0.19 \pm 0.37 \pm 0.01) \times 10^{-4}$

where the errors are statistical, systematic and the uncertainty arising from translating from the center-of-mass frame to the $B$-meson rest frame. The value of the lower energy cut, $1.7 \mathrm{GeV}$, is the lowest achieved thus far in a measurement of inclusive $B \rightarrow X_{s} \gamma$. These results are preliminary.

\section{Analysis of $B \rightarrow K^{(*)} \ell^{+} \ell^{-}$}

The decays $B \rightarrow K^{(*)} \ell^{+} \ell^{-}$are exclusives modes of the $b \rightarrow s \ell^{+} \ell^{-}$process, which is analogous to the $b \rightarrow s \gamma$ decay, with the photon producing a lepton pair. In the $b \rightarrow s \ell^{+} \ell^{-}$case, however, there are electroweak contributions, with the $Z^{0}$ boson replacing the photon, plus an additional "box" diagram involving $W^{ \pm}$bosons. The contributing amplitudes are expressed in terms of

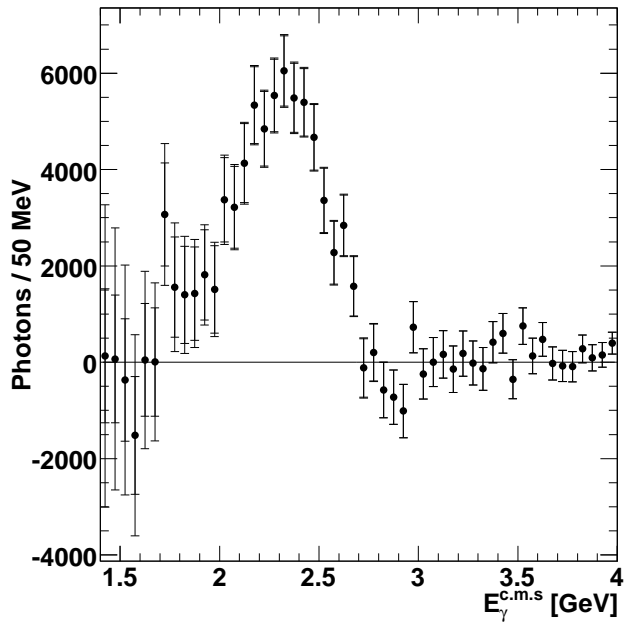

Figure 2. The extracted photon energy spectrum for Belle's measurement $B \rightarrow X_{s, d} \gamma$. The two error bars show the statistical and total errors.

hadronic form factors and effective Wilson coefficients $C_{7}^{\text {eff }}, C_{9}^{\text {eff }}$ and $C_{10}^{\text {eff }}$, corresponding to the electromagnetic penguin diagram, and the vector and axial-vector part of the $Z^{0}$ and $W^{+} W^{-}$box diagrams, respectively [7].

The rate for $b \rightarrow s \ell^{+} \ell^{-}$is quite small and experimental efforts have mostly been expended on studying exclusive states, where the background can be kept to a low level. This means that branching fraction measurements become less important, since the uncertainties on the SM predictions for exclusive $B \rightarrow K^{(*)} \ell^{+} \ell^{-}$are on the order of $30 \%$.

The presence of the lepton pair in the final state gives rise to new observables, compared to $B \rightarrow X_{s} \gamma$, such as the forward-backward lepton asymmetry and the $K^{*}$ polarization fraction. Additional quantities that are sensitive to NP, such as the direct $\mathrm{CP}$ and isospin asymmetries and the lepton flavour ratio, tend to have more precise SM predictions than the branching fractions, because some uncertainties cancel when taking ratios. In general, the effect of NP on these quantities will be a function of $q^{2} \equiv m_{\ell^{+} \ell^{-}}^{2}$, and measurements are performed in bins of $q^{2}$ where possible. 
Recent measurements of these quantities have been performed at BABAR [9]10]. The following preliminary results are based on a data sample of 384 million $B \bar{B}$ pairs.

The decays $B \rightarrow K^{(*)} \ell^{+} \ell^{-}$are reconstructed in ten different final states containing an $e^{+} e^{-}$or $\mu^{+} \mu^{-}$pair, together with a $K_{s}^{0}, K^{+}$or $K^{*}(892)$ candidate, where $K^{*}(892)$ is reconstructed in the $K^{+} \pi^{-}, K^{+} \pi^{0}$ and $K_{s}^{0} \pi^{+}$modes. The primary backgrounds from semileptonic decays of $B$ and $D$ mesons are suppressed using multivariate techniques, while events containing $J / \psi$ or $\psi^{\prime}$ decays are vetoed explicitly.

The events are divided into a low $q^{2}$ region $\left(0.1<q^{2}<7.02 \mathrm{GeV}^{2} / \mathrm{c}^{4}\right)$ and a high $q^{2}$ region $\left(q^{2}>10.24 \mathrm{GeV}^{2} / \mathrm{c}^{4}\right)$.

$B$-meson candidates are identified using the kinematic variables $m_{E S}=\sqrt{s / 4-p_{B}^{* 2}}$ and $\Delta E=E_{B}^{*}-\sqrt{s} / 2$, where $p_{B}^{*}$ and $E_{B}^{*}$ are the $B$ momentum and energy in the $\Upsilon(4 S)$ center-ofmass (CM) frame, and $\sqrt{s}$ is the total CM energy. Typically, a selection is made on $\Delta E$ (either \pm 40 $\mathrm{MeV}$ or $\pm 50 \mathrm{MeV}$, depending on the $q^{2}$ region) and a fit to $m_{E S}$ is used to extract the signal yield.

Example $m_{E S}$ fits are depicted in Fig. 3, which shows the distributions for neutral and charged $K$ and $K^{*}$ channels in the low- $q^{2}$ region.

\subsection{Direct CP, Isospin and Lepton Flavour Asymmetries}

The extraction of event yields described above allows the measurement of several asymmetries that are sensitive to the presence of New Physics.

The direct CP asymmetry

$$
A_{C P} \equiv \frac{\mathcal{B}\left(\bar{B} \rightarrow \bar{K}^{(*)} \ell^{+} \ell^{-}\right)-\mathcal{B}\left(B \rightarrow K^{(*)} \ell^{+} \ell^{-}\right)}{\mathcal{B}\left(\bar{B} \rightarrow \bar{K}^{(*)} \ell^{+} \ell^{-}\right)+\mathcal{B}\left(B \rightarrow K^{(*)} \ell^{+} \ell^{-}\right)}
$$

is expected to be $O\left(10^{-3}\right)$ in the SM, but new physics at the electroweak scale could produce a significant enhancement [11. The results found for this analysis are reported in Table 1. We find no evidence for a deviation from the Standard Model.

In the Standard Model the ratio of rates to dimuon and di-electron final states

$R_{K^{(*)}} \equiv \frac{\mathcal{B}\left(K^{(*)} \mu^{+} \mu^{-}\right)}{\mathcal{B}\left(K^{(*)} e^{+} e^{-}\right)}$

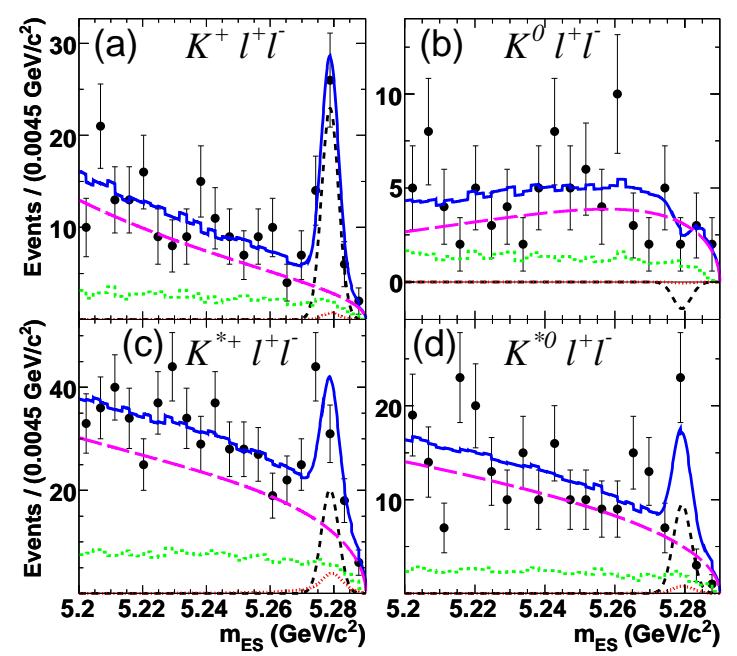

Figure 3. Distributions of $m_{E S}$ with fit results in the low $q^{2}$ region for the $B A B A R B \rightarrow$ $K^{(*)} \ell^{+} \ell^{-}$analysis. Total fit [solid], combinatoric background [long dash], signal [medium dash], hadronic background [short dash], peaking background [dots].

is very close to one [12. In some NP models, such as supersymmetry, this ratio can be altered significantly. Deviations from unity as large as $\sim 10 \%$ are possible for large values of $\tan \beta[13$.

In the region $q^{2}<\left(2 m_{\mu}\right)^{2}$, where only the $e^{+} e^{-}$ modes are allowed, there is a large enhancement of $K^{*} e^{+} e^{-}$due to a $1 / q^{2}$ scaling of the photon penguin. The expected SM value of $R_{K^{*}}$ including this region is 0.75 [12] The results for $R_{K^{(*)}}$,

Table 1

$A_{C P}$ results from $B A B A R$. The high $q^{2}$ region, not shown here for lack of space, appears in [9]. The quoted errors are statistical and systematic, respectively.

\begin{tabular}{lcc}
\hline \hline Mode & combined $q^{2}$ & low $q^{2}$ \\
\hline$K^{+} \ell^{+} \ell^{-}$ & $-0.18_{-0.18}^{+0.18} \pm 0.01$ & $-0.18_{-0.19}^{+0.19} \pm 0.01$ \\
$K^{* 0} \ell^{+} \ell^{-}$ & $0.02_{-0.20}^{+0.20} \pm 0.02$ & $-0.23_{-0.38}^{+0.38} \pm 0.02$ \\
$K^{*+} \ell^{+} \ell^{-}$ & $0.01_{-0.24}^{+0.26} \pm 0.02$ & $0.10_{-0.24}^{+0.25} \pm 0.02$ \\
$K^{*} \ell^{+} \ell^{-}$ & $0.01_{-0.15}^{+0.16} \pm 0.01$ & $0.01_{-0.20}^{+0.21} \pm 0.01$ \\
\hline \hline
\end{tabular}


Table 2

$R_{K^{(*)}}$ results in each $q^{2}$ region. The extended ("ext.") regions are relevant only for $R_{K^{*}}$. The errors are statistical and systematic, respectively.

\begin{tabular}{lcc}
\hline \hline$q^{2}$ Region & $R_{K^{*}}$ & $R_{K}$ \\
\hline combined & $1.37_{-0.40}^{+0.53} \pm 0.09$ & $0.96_{-0.34}^{+0.44} \pm 0.05$ \\
ext. comb. & $1.10_{-0.32}^{+0.42} \pm 0.07$ & - \\
low & $1.01_{-0.44}^{+0.58} \pm 0.08$ & $0.40_{-0.23}^{+0.30} \pm 0.02$ \\
ext. low & $0.56_{-0.23}^{+0.29} \pm 0.04$ & $-\overline{-1.04}$ \\
high & $2.15_{-0.78}^{+1.42} \pm 0.15$ & $1.06_{-0.51}^{+0.81} \pm 0.06$ \\
\hline \hline
\end{tabular}

shown in Table 2, indicate no deviation from the Standard Model.

The CP-averaged isospin asymmetry:

$$
\begin{aligned}
& A_{I}^{K^{(*)}} \equiv \\
& \frac{\mathcal{B}\left(B^{0} \rightarrow K^{(*) 0} \ell^{+} \ell^{-}\right)-r \mathcal{B}\left(B^{ \pm} \rightarrow K^{(*) \pm} \ell^{+} \ell^{-}\right)}{\mathcal{B}\left(B^{0} \rightarrow K^{(*) 0} \ell^{+} \ell^{-}\right)+r \mathcal{B}\left(B^{ \pm} \rightarrow K^{(*) \pm} \ell^{+} \ell^{-}\right)},
\end{aligned}
$$

where $r=\tau_{0} / \tau_{+}=1 /(1.07 \pm 0.01)$ is the ratio of the $B^{0}$ and $B^{+}$lifetimes [14, has a SM expectation of $6-13 \%$ as $q^{2} \rightarrow 0 \mathrm{GeV}^{2}$ [15]. A calculation of the predicted $K^{*+}$ and $K^{* 0}$ rates integrated over the low $q^{2}$ region gives $A_{I}^{K^{*}}=$ $-0.005 \pm 0.020$ [1617.

The results for the isospin asymmetry are presented in Table 3 While we find no significant asymmetry in the high or combined $q^{2}$ regions, we do measure a large asymmetry in the low $q^{2}$ region. The origin of this non-zero asymmetry can be observed in Fig. 3 above, where fewer events are found in the neutral modes compared to the charged modes. Combining all modes in the low $q^{2}$ region, we find $A_{I}^{K^{(*)}}=-0.64_{-0.14}^{+0.15} \pm 0.03$. Including systematics, this is a $3.9 \sigma$ difference from a null $A_{I}^{K^{(*)}}$ hypothesis.

This large isospin asymmetry is unexpected in the Standard Model, which predicts essentially zero asymmetry integrated over the low $q^{2}$ region.

These results are preliminary.

\subsection{Angular analysis of $K^{*} \ell^{+} \ell^{-}$decays}

It is also possible to probe the effects of New Physics by analyzing the angular distributions of the decay products in $K^{*} \ell^{+} \ell^{-}$decays as a function of $q^{2}$ [18. In particular, the $K^{*}$ polarization fraction can be determined from the distribution
Table 3

$A_{I}^{K^{(*)}}$ results from BABAR. The high $q^{2}$ region, not shown here for lack of space, appears in [9]. The errors are statistical and systematic, respectively. The last table row shows $K^{*} e^{+} e^{-}$results for the extended regions.

\begin{tabular}{lcc}
\hline \hline Mode & combined $q^{2}$ & low $q^{2}$ \\
\hline$K \mu^{+} \mu^{-}$ & $0.13_{-0.37}^{+0.29} \pm 0.04$ & $-0.91_{-\infty}^{+1.2} \pm 0.18$ \\
$K e^{+} e^{-}$ & $-0.73_{-0.50}^{+0.39} \pm 0.04$ & $-1.41_{-0.69}^{+0.49} \pm 0.04$ \\
$K \ell^{+} \ell^{-}$ & $-0.37_{-0.34}^{+0.27} \pm 0.04$ & $-1.43_{-0.85}^{+0.56} \pm 0.05$ \\
$K^{*} \mu^{+} \mu^{-}$ & $-0.00_{-0.26}^{+0.36} \pm 0.05$ & $-0.26_{-0.34}^{+0.50} \pm 0.05$ \\
$K^{*} e^{+} e^{-}$ & $-0.20_{-0.20}^{+0.22} \pm 0.03$ & $-0.66_{-0.17}^{+0.19} \pm 0.02$ \\
$K^{*} \ell^{+} \ell^{-}$ & $-0.12_{-0.16}^{+0.18} \pm 0.04$ & $-0.56_{-0.15}^{+0.17} \pm 0.03$ \\
\hline$K^{*} e^{+} e^{-}$ & $-0.27_{-0.18}^{+0.21} \pm 0.03$ & $-0.25_{-0.18}^{+0.20} \pm 0.03$ \\
\hline \hline
\end{tabular}

of the angle $\theta_{K}$

$\frac{3}{2} F_{L} \cos ^{2} \theta_{K}+\frac{3}{4}\left(1-F_{L}\right)\left(1-\cos ^{2} \theta_{K}\right)$

where $\theta_{K}$ is the angle between the $K$ and $B$ directions in the $K^{*}$ rest frame.

Furthermore, the lepton forward-backward asymmetry, $A_{F B}$, can be determined from the distribution of the angle $\theta_{\ell}$

$$
\begin{aligned}
& \frac{3}{4} F_{L}\left(1-\cos ^{2} \theta_{\ell}\right) \\
& \quad+\frac{3}{8}\left(1-F_{L}\right)\left(1+\cos ^{2} \theta_{\ell}\right)+A_{F B} \cos \theta_{\ell} .
\end{aligned}
$$

Here $\theta_{\ell}$ is the angle between the $\ell^{+}\left(\ell^{-}\right)$and the $B(\bar{B})$ direction in the $\ell^{+} \ell^{-}$rest frame.

Fits to the $K^{*} \ell^{+} \ell^{-}$sample are shown in Fig. 4 and the extracted values of $F_{L}$ and $A_{F B}$ are shown in Table 4 and plotted in Fig. 5 . Results are consistent with the Standard Model, and the $A_{F B}$ results exclude a wrong-sign $C_{9} C_{10}$ from purely right-handed weak currents at $3 \sigma$ significance.

These results are preliminary.

\section{Search for $B \rightarrow \pi \ell^{+} \ell^{-}$}

The rare mode $B \rightarrow \pi \ell^{+} \ell^{-}$is the $b \rightarrow d \gamma$ analogue to the $B \rightarrow K \ell^{+} \ell^{-}$decay. The Standard Model rate for $B \rightarrow \pi \ell^{+} \ell^{-}$is thus suppressed relative to the $B \rightarrow K^{+} \ell^{-}$mode by the factor $\left|V_{t d} / V_{t s}\right|^{2} \sim 0.04$; the SM predictions for the 

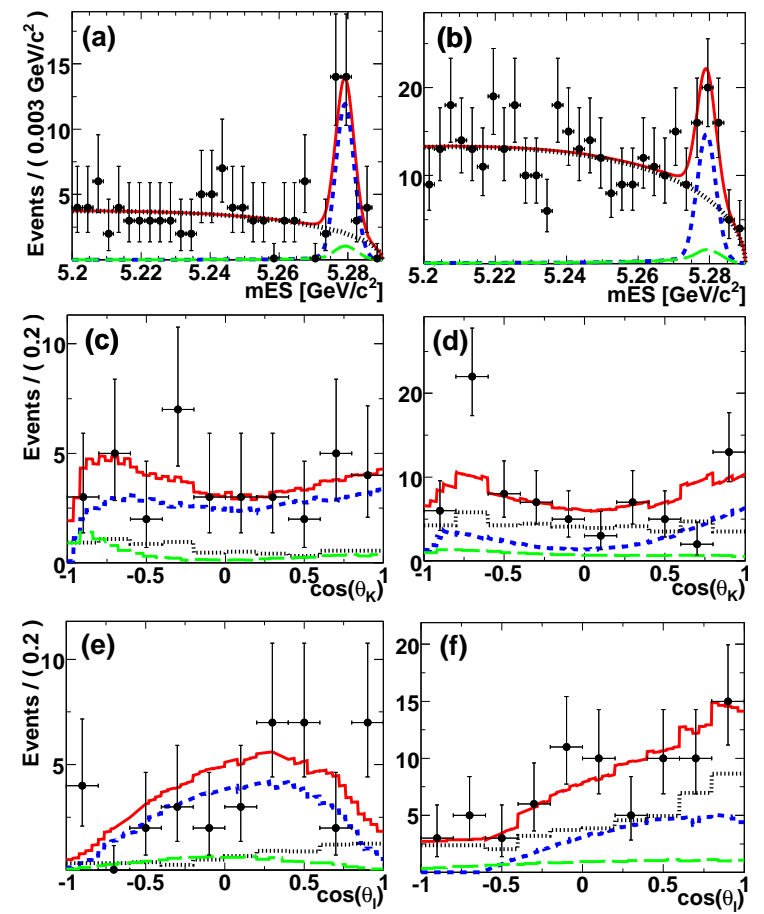

Figure 4. $K^{*} \ell^{+} \ell^{-}$fits from BABAR: (a) low $q^{2} m_{E S}$, (b) high $q^{2} m_{E S}$, (c) low $q^{2} \cos \theta_{K}$, (d) high $q^{2} \cos \theta_{K}$, (e) low $q^{2} \cos \theta_{\ell}$, (f) high $q^{2} \cos \theta_{\ell}$; with combinatorial (dots) and peaking (long dash) background, signal (short dash) and total (solid) fit distributions superimposed on the data points.

Table 4

Results for the fits to the $K \ell^{+} \ell^{-}$and $K^{*} \ell^{+} \ell^{-}$ samples. $N_{S}$ is the number of signal events in the $m_{E S}$ fit. The quoted errors are statistical only.

\begin{tabular}{lcccc}
\hline \hline Decay & $q^{2}$ & $N_{S}$ & $F_{L}$ & $A_{F B}$ \\
\hline$K \ell^{+} \ell^{-}$ & low & $26.0 \pm 5.7$ & & $+0.04_{-0.24}^{+0.16}$ \\
& high & $26.5 \pm 6.7$ & & $+0.20_{-0.22}^{+0.14}$ \\
\hline$K^{*} \ell^{+} \ell^{-}$ & low & $27.2 \pm 6.3$ & $0.35 \pm 0.16$ & $+0.24_{-0.23}^{+0.18}$ \\
& high & $36.6 \pm 9.6$ & $0.71_{-0.22}^{+0.20}$ & $+0.76_{-0.32}^{+0.52}$ \\
\hline \hline
\end{tabular}

branching fractions are $3.3 \times 10^{-8}$ and $1.7 \times 10^{-8}$ for the charged and neutral modes, respectively.

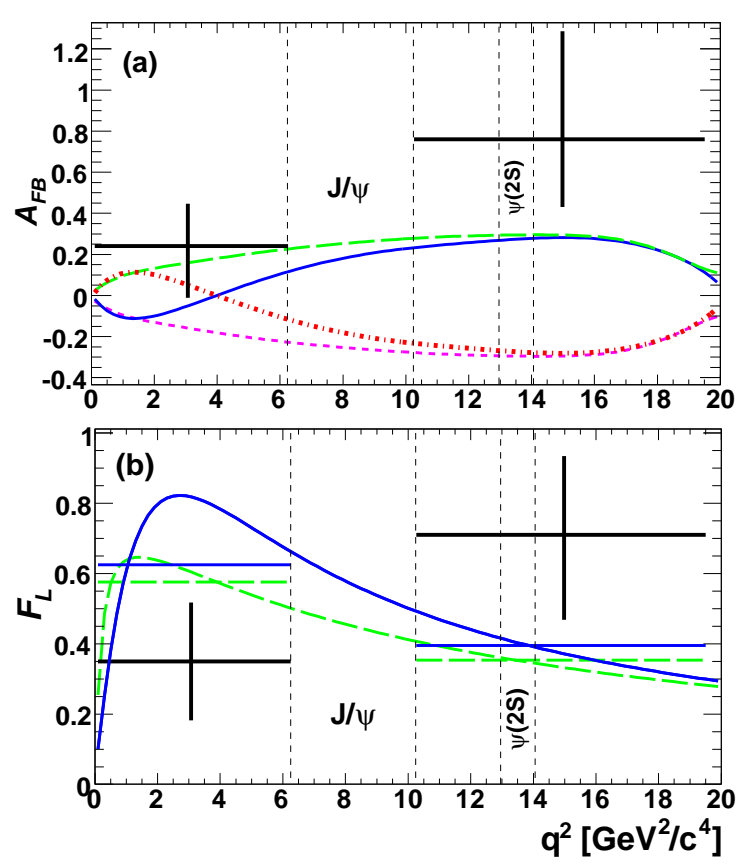

Figure 5. Plots of BABAR's results for (a) $A_{F B}$ and (b) $F_{L}$ for the decay $B \rightarrow K^{*} \ell^{+} \ell^{-}$showing comparisons with $\mathrm{SM}$ (solid); $C_{7}=-C_{7}^{S M}$ (long dash); $C_{9} C_{10}=-C_{9}^{S M} C_{10}^{S M}$ (short dash); $C_{7}=-C_{7}^{S M}, C_{9} C_{10}=-C_{9}^{S M} C_{10}^{S M}$ (dash-dot). Statistical and systematic errors are added in quadrature. Expected $F_{L}$ values integrated over each $q^{2}$ region are also shown. The $F_{L}$ curves with $C_{9} C_{10}=-C_{9}^{S M} C_{10}^{S M}$ are nearly identical to the two curves shown.

Belle has recently performed a search for $B \rightarrow$ $\pi \ell^{+} \ell^{-}$on a data sample of $605 \mathrm{fb}^{-1}$, reconstructing both the neutral and charged modes, with $\ell$ being $\mu$ or $e$ [19]. A Fisher discriminant using 16 event shape variables suppresses background from the continuum, while events containing $J / \psi$ or $\psi^{\prime}$ decays are vetoed explicitly. A fit to the beamconstrained mass is used to extract the yields.

No significant signal was found. These preliminary results, shown in Table 5, indicate that current sensitivity is approaching the Standard Model prediction for the charged mode, although there is still a ways to go for the neutral mode. 
Table 5

A summary of the signal yields $\left(N_{s}\right)$, reconstruction efficiencies $(\epsilon)$, and $90 \%$ confidence level upper limits (U.L.) for $B \rightarrow \pi \ell^{+} \ell^{-}$.

\begin{tabular}{lccc}
\hline Mode & $N_{s}$ & $\epsilon(\%)$ & U.L. $\left(10^{-8}\right)$ \\
\hline$B^{+} \rightarrow \pi^{+} \mu^{+} \mu^{-}$ & $0.5_{-1.9}^{+2.8}$ & 13.1 & 6.9 \\
$B^{+} \rightarrow \pi^{+} e^{+} e^{-}$ & $1.4_{-2.3}^{+3.2}$ & 13.8 & 8.0 \\
$B^{+} \rightarrow \pi^{+} \ell^{+} \ell^{-}$ & - & - & 4.9 \\
\hline$B^{0} \rightarrow \pi^{0} \mu^{+} \mu^{-}$ & $5.1_{-3.3}^{+4.2}$ & 9.6 & 18.4 \\
$B^{0} \rightarrow \pi^{0} e^{+} e^{-}$ & $2.7_{-4.0}^{+5.2}$ & 7.4 & 22.7 \\
$B^{0} \rightarrow \pi^{0} \ell^{+} \ell^{-}$ & - & - & 15.4 \\
\hline$B \rightarrow \pi \ell^{+} \ell^{-}$ & - & - & 6.2 \\
\hline
\end{tabular}

\section{Searches for $B \rightarrow K^{(*)} \nu \bar{\nu}$}

The mode $B \rightarrow K^{(*)} \nu \bar{\nu}$ is similar to the $B \rightarrow K^{(*)} \ell^{+} \ell^{-}$mode, but with the charged lepton pair replaced by a pair of neutrinos. Since the neutrinos are not detected experimentally, $B \rightarrow K^{*} \ell^{+} \ell^{-}$observables such as $A_{F B}$ are not measurable and the focus on this channel has been to measure its branching fraction.

The SM branching fractions are predicted to be $\left(3.8_{-0.6}^{+1.2}\right) \times 10^{-6}$ and $\left(1.3_{-0.3}^{+0.4}\right) \times 10^{-5}$ for the $K \nu \bar{\nu}$ and $K^{*} \nu \bar{\nu}$ channels, respectively 20 . These are larger than for the corresponding $B \rightarrow K^{(*)} \ell^{+} \ell^{-}$ modes, but the missing neutrinos present considerable experimental difficulties, leading to lower sensitivities for this channel. The neutrinos' momentum is typically inferred by reconstructing all the other particles in the event. When the other $B$-meson is fully reconstructed, the signal decay yields a single charged track (or $K^{*}$ candidate) and significant missing energy.

$B A B A R$ has recently performed searches for $B \rightarrow K \nu \bar{\nu}$ and $B \rightarrow K^{*} \nu \bar{\nu}$ reconstructing the other $B$-meson in the event in a semileptonic decay mode, $B \rightarrow D \ell \nu(X)$. Multivariate discriminators are used to reduce the background. For the $B \rightarrow K^{*} \nu \bar{\nu}$ mode, a variable $E_{\text {extra }}$ is constructed: it is the sum of the leftover energy in the event, excluding the particles used to reconstruct the tag-side $B$-meson and the signal $K^{*}$ candidate. An excess of events at $E_{\text {extra }} \sim 0$ would be an indication of $B \rightarrow K^{*} \nu \bar{\nu}$ decays. For the
$B \rightarrow K \nu \bar{\nu}$ channel, the multivariate discriminator is used to isolate the signal. For both channels, there is no evidence of a significant signal and the derived $90 \%$ confidence level upper limits are found to be

$$
\begin{aligned}
\mathcal{B}\left(B^{+} \rightarrow K^{*+} \nu \bar{\nu}\right) & <9 \times 10^{-5} \\
\mathcal{B}\left(B^{0} \rightarrow K^{* 0} \nu \bar{\nu}\right) & <21 \times 10^{-5} \\
\mathcal{B}\left(B^{+} \rightarrow K^{+} \nu \bar{\nu}\right) & <4.2 \times 10^{-5}
\end{aligned}
$$

The limits for $B \rightarrow K^{*} \nu \bar{\nu}$ are better than previously report results 21, although we are still fairly far from the SM prediction.

\section{Status of $B \rightarrow \tau \nu$}

The $B \rightarrow \tau \nu$ branching fraction is given in the Standard Model by

$\mathcal{B}\left(B^{+} \rightarrow \tau^{+} \nu\right)=\frac{G_{F}^{2} m_{B} m_{\tau}^{2}}{8 \pi}\left[1-\frac{m_{\tau}^{2}}{m_{B}^{2}}\right]^{2} \tau_{B^{+}} f_{B}^{2}\left|V_{u b}\right|^{2}$,

where $G_{F}$ is the Fermi constant, $\tau_{B^{+}}$is the $B^{+}$ lifetime and $m_{B}$ and $m_{\tau}$ are the $B^{+}$meson and $\tau$ lepton masses. The branching fraction can be significantly modified by New Physics containing a charged Higgs boson. In minimal supersymmetric models, for example, the SM branching fraction is modified [22]:

$$
\begin{aligned}
& \mathcal{B}\left(B^{+} \rightarrow \tau^{+} \nu\right)= \\
& \mathcal{B}\left(B^{+} \rightarrow \tau^{+} \nu\right)_{S M}\left[1-\tan ^{2} \beta \frac{m_{B^{+}}^{2}}{m_{H^{+}}^{2}}\right]^{2} .
\end{aligned}
$$

Belle and $B A B A R$ have measured the branching fraction of $B \rightarrow \tau \nu$ 23|2425]. The experimental techniques are similar to the $B \rightarrow K^{(*)} \nu \bar{\nu}$ analyses: reconstruction of the tag $B$-meson in either a semileptonic or hadronic decay mode, multivariate discriminators to further suppress backgrounds and construction of an $E_{\text {extra }}$ variable, which represents the energy left over after excluding daughters of the tag $B$-meson and the $\tau$ lepton, reconstructed in one of: $\mu^{+} \nu \bar{\nu}, e^{+} \nu \bar{\nu}$, $\pi^{+} \nu \bar{\nu}$ or $\pi^{+} \pi^{0} \nu \bar{\nu}$. An excess near $E_{\text {extra }} \sim 0$ is a signature of $B \rightarrow \tau \nu$ decays.

Results are shown in Table 6. While no single measurement is highly significant, the average is significant and consistent with the Standard Model expectation. 
Table 6

Results for $B \rightarrow \tau \nu$. The uncertainties on the branching fractions are statistical and systematic.

\begin{tabular}{lcc}
\hline Experiment & $\begin{array}{c}\text { Tag } \\
\text { Mode }\end{array}$ & $\begin{array}{c}\text { Branching } \\
\text { Fraction }\end{array}$ \\
\hline BaBar [23] & had & $(1.8 \pm 0.9 \pm 0.5) \times 10^{-4}$ \\
BaBar [24] & SL & $(0.9 \pm 0.6 \pm 0.1) \times 10^{-4}$ \\
Belle [25] & had & $(1.8 \pm 0.5 \pm 0.5) \times 10^{-4}$ \\
\hline HFAG Average & - & $(1.41 \pm 0.42) \times 10^{-4}$ \\
\hline
\end{tabular}

\section{Conclusions}

Radiative penguin and leptonic $B$-meson decays are excellent probes for investigating the effects of New Physics. Although current measurements are in agreement with the Standard Model expectations, they are still quite useful for setting bounds on possible NP models. The $B \rightarrow X_{s} \gamma$ and $B \rightarrow \tau \nu$ measurements, for example, put strong constraints on the mass of charged Higgs bosons in Type II two-Higgs double models [26]. The $B \rightarrow X_{s} \gamma$ branching fraction measurements also constrain models with universal extra dimensions [27].

Finally, I would like to express my heartfelt thanks to the conference organizers for a stimulating conference and warm hospitality.

\section{REFERENCES}

1. B. Aubert et al. [BABAR Collaboration], Nucl. Instrum. Meth. A 479, 1 (2002).

2. A. Abashian et al. the Belle Collaboration, Nucl. Instrum. Methods Phys. Res., Sect. A 479, 117 (2002).

3. M. Misiak et al., Phys. Rev. Lett. 98, 022002 (2007); T. Becher and M. Neubert, Phys. Rev. Lett. 98, 022003 (2007).

4. B. Aubert et al. the BABAR Collaboration, Phys. Rev. D 77, 051103(R) (2008).

5. B. Aubert et al. the BABAR Collaboration, Phys. Rev. Lett. 97, 171803 (2006).

6. K. Abe et al. the Belle Collaboration, arXiv:0804.1580 [hep-ex].

7. G. Buchalla, A. J. Buras and M. E. Lautenbacher, Rev. Mod. Phys. 68, 1125 (1996).
8. A. Ali et al., Phys. Rev. D 66, 034002 (2002).

9. B. Aubert et al. the BABAR Collaboration, arXiv:0807.4119 [hep-ex]; submitted to Phys. Rev. Lett.

10. B. Aubert et al. the BABAR Collaboration, arXiv:0804.4412 [hep-ex]; submitted to Phys. Rev. Lett.

11. C. Bobeth, G. Hiller and G. Piranishvili, arXiv:0805.2525 (2008).

12. G. Hiller and F. Kruger, Phys. Rev. D 69, 074020 (2004).

13. Q. S. Yan, C. S. Huang, W. Liao and S. H. Zhu, Phys. Rev. D 62, 094023 (2000).

14. Heavy Flavor Averaging Group, E. Barberio et al., arXiv:0704.3575 (2007).

15. T. Feldmann and J. Matias, JHEP 0301, 074 (2003).

16. M. Beneke, T. Feldmann and D. Seidel Eur. Phys. J. C41, 173 (2005).

17. T. Feldmann, private communication.

18. G. Burdman, Phys. Rev. D 52, 6400 (1995); J. L. Hewett and J. D. Wells, Phys. Rev. D 55, 5549 (1997); T. Feldmann and J. Matias, JHEP 0301, 074 (2003); W. J. Li, Y. B. Dai and C. S. Huang, Eur. Phys. J. C 40, 565 (2005); Y. G. Xu, R. M. Wang and Y. D. Yang, Phys. Rev. D 74, 114019 (2006); P. Colangelo, F. De Fazio, R. Ferrandes and T. N. Pham, Phys. Rev. D 73, 115006 (2006).

19. K. Abe et al. the Belle Collaboration, arXiv:0804.3656 [hep-ex], accepted by Phys. Rev. D.

20. B. Buchalla, G. Hiller and G. Isidori, Phys. Rev. D 63, 014015 (2001).

21. K. Abe et al. the Belle Collaboration, Phys. Rev. Lett. 99, 221802 (2007).

22. W. Hou, Phys. Rev. D 48, 2342 (1993).

23. B. Aubert et al. the BABAR Collaboration, Phys. Rev. D 77, 011107 (2008).

24. B. Aubert et al. the BABAR Collaboration, Phys. Rev. D 77, 052002 (2007).

25. K. Abe et al. the Belle Collaboration, Phys. Rev. Lett. 97, 251802 (2006).

26. U. Haisch, arXiv:0805.2141 [hep-ph].

27. A. Freitas and U. Haisch, arXiv:0801.4446 [hep-ph]. 